ISSN 1823-626X

Malaysian Journal of Fundamental and Applied Sciences

available online at http://mjfas.ibnusina.utm.my

\title{
Spatial interaction of Electroencephalography signal during epileptic seizure on Flat Electroencephalography
}

\author{
Goh Chien Yong ${ }^{1,2 *}$, Tahir Ahmad ${ }^{1,2}$ and Normah Maan ${ }^{2}$ \\ ${ }^{1}$ Ibnu Sina Institute for Fundamental Science Studies, Universiti Teknologi Malaysia, 81310 Skudai, Johor, Malaysia \\ ${ }^{2}$ Department of Mathematic Sciences, Faculty Science, Universiti Teknologi Malaysia, 81310 Skudai, Johor, Malaysia \\ Received 12 January 2013, Revised 3 April 2013, Accepted 25 May 2013, Available online 2 June 2013
}

\begin{abstract}
Electroencephalography (EEG) is one of the fields in clinical neurophysiology, which is a recording of the electrical activity of the brain from the scalp. One of the major roles of EEG is as an aid to diagnose epilepsy. Abnormal patterns such as spikes, sharp waves and, spikes and wave complexes can be seen. It is important to extract spatial information about the dynamics from a few observations of this recorded signal regardless where EEG sensors are located. A developed method by Theoretical \& Computational Modelling for Complex System (TCM), UTM, namely Flat EEG for mapping high dimensional signal into a low dimensional space will be used as platforms to analyse EEG signal spatially during epileptic seizure. The spatial interactions among clusters are identified through spatial interaction model, namely gravity model. This paper also reveals that gravity model used is a measure.
\end{abstract}

| EEG| Epileptic| Flat EEG| Spatial interaction | Gravity model| Measure|

(B) 2013 Ibnu Sina Institute. All rights reserved. http://dx.doi.org/10.11113/mjfas.v9n4.110

\section{INTRODUCTION}

Epilepsy is the second most common neurological disorder, affecting $1 \%$ of world population [1]. Sanei and Chambers (2007) defined epilepsy as a sudden and recurrent brain malfunction and a disease that reflects an excessive and hypersynchronous activity of the neurons within the brain [2]. The paper indicated that it is the most prevalent brain disorder among adults and children, second only to stroke. In this case the seizures may occur randomly and they impair the normal function of the brain. There are many treatments that can be applied on epilepsy. Pharmacological is the most important treatment among all today, followed by surgical treatments. However, a successful surgical treatment of focus epilepsies has a major requirement, which is exact localization of the epileptic focus and its delineation from functionally relevant areas. Electroencephalogram (EEG) is a recording of the electrical activity of the brain from the scalp. It plays an important diagnostic role in epilepsy and provides supporting evidence of a seizure disorder as well as assisting with classification of seizures and epilepsy syndromes. In epilepsy there is a miniature brainstorm of certain groups of brain cells that can lead to convulsions. This virtual explosion of electricity can be detected on an EEG used subsequently by neurologists to locate where damaged brain tissue lies. That's why EEG remains the most useful and cost effective modality for the study of epilepsy.
Most traditional epilepsy analysis methods, based on the EEG, are focused on the detection and classification of epilepsy seizures, among which the best method of analysis is still the visual inspection of EEG by a highly skilled electroencephalographers or neurophysiologist [2].

This paper is organized into four sections. In the next section provides some literature review on Flat EEG, measure and spatial interaction. In section 3, the construction and definition of our model is carried out. We prove the intended model is a measure and the implementation of the model is presented. Finally, we draw some conclusions in the last section of this paper.

\section{LITERATURE REVIEW}

\subsection{Flat EEG}

In 2007, Fauziah worked on the method of producing 'Flat EEG' where these signals can be compressed and analysed [3]. It is a new method for mapping high dimensional signal, namely EEG into a low dimensional space. Fauziah's EEG coordinate system (Fig. 1a) is defined as

$$
\begin{aligned}
& C_{E E G} \\
& =\left\{\left((\mathrm{x}, \mathrm{y}, \mathrm{z}), \mathrm{e}_{\mathrm{p}}\right): \mathrm{x}, \mathrm{y}, \mathrm{z}, \mathrm{e}_{\mathrm{p}} \in \mathbb{R} \text { and } \mathrm{x}^{2}+\mathrm{y}^{2}+\mathrm{z}^{2}=\mathrm{r}^{2}\right\}
\end{aligned}
$$

where $r$ is the radius of a patient head. 
Furthermore, the mapping of $\mathrm{C}_{\mathrm{EEG}}$ to a plane is defined as follows:

$\mathrm{S}_{\mathrm{t}}: \mathrm{C}_{\mathrm{EEG}} \rightarrow \mathrm{MC}$ (see Fig. 1b) such that

$\mathrm{S}_{\mathrm{t}}\left((\mathrm{x}, \mathrm{y}, \mathrm{z}), \mathrm{e}_{\mathrm{p}}\right)=\left(\frac{\mathrm{rx}+\text { iry }}{\mathrm{r}+\mathrm{z}}, \mathrm{e}_{\mathrm{p}}\right)=\left(\frac{\mathrm{rx}}{\mathrm{r}+\mathrm{z}}, \frac{\mathrm{ry}}{\mathrm{r}+\mathrm{z}}\right)_{\mathrm{e}_{\mathrm{p}},(\mathrm{x}, \mathrm{y}, \mathrm{z})}$ where $M C=\left((x, y), e_{p}\right): x, y, e_{p} \in \mathbb{R}$

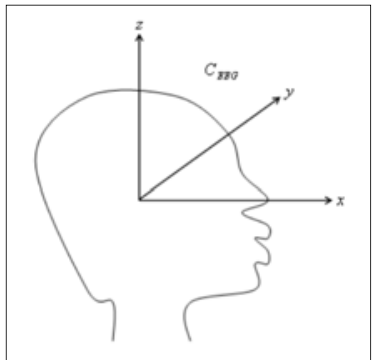

(a) EEG Coordinate System.

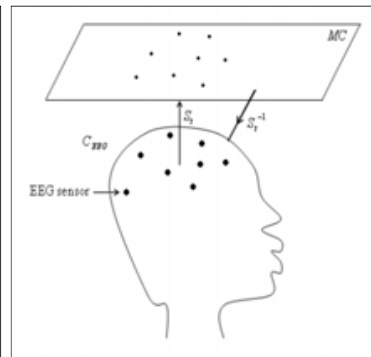

(b) EEG Projection.
Fig. 1

is the first component of FTTM [4]. Both $C_{E E G}$ and MC were designed and proven as 2-manifolds [5].

Meanwhile $S_{t}$ is designed to be a one to one function as well as being conformal. Details of proofs contain in [3]. The EEG signal during seizure as Figure 2 can be compressed to Figure 3 (a) and analysed second by second as Figure 3 (b) $[3,6]$. The flattening method has been coded as a software and received copyright (C) 2006 Universiti Teknologi Malaysia - All Rights Reserved.

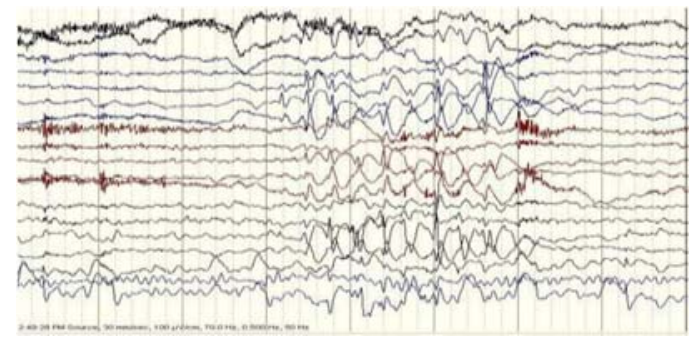

Fig. 2 EEG signal.

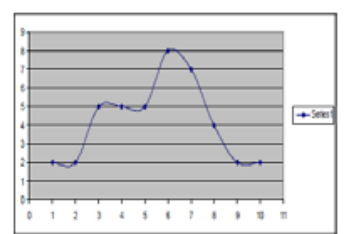

(a)

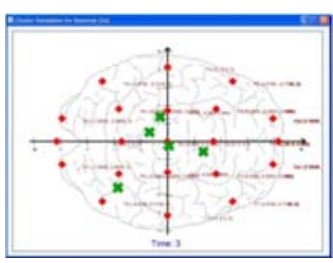

(b)
Fig. 3 Flat EEG

\subsection{Measure}

The given following definitions are primarily used in the proving that our intended model is a measure.
Definition 1 (Ring) [7]: A ring is a nonempty set $\mathrm{R}$ together with two binary operations (usually denoted as addition $(+)$ and multiplication) such that:

(1) $(\mathcal{R},+)$ is an abelian group;

(2) $a(b c)=(a b) c$ for all $a, b, c \in \mathcal{R}$ (associative multiplication);

(3) $a(b+c)=a b+a c$ and $(a+b) c=a c+b c$ (left and right distributive laws).

Definition 2 (Ring of sets) [8]: A family $\mathcal{R}$ of sets is called a ring if $A \in \mathcal{R}$ and $B \in \mathcal{R}$ implies:

(1) $A \cup B \in \mathcal{R}$

(2) $A \cap B \in \mathcal{R}$.

Note that since a ring of sets can be an empty set. Some authors define a ring of sets to be a non-empty set with second condition above replaced by: $A-B \in \mathcal{R}$ [8].

Definition 3 (Measure) [8]: A measure is a set function $\mu$ whose domain of definition is a ring $\mathcal{R}$, such that :

(1) $\mu$ is additive.

(2) $\mu$ is positive.

(3) $\mu(\varnothing)=0$.

(4) If $E_{n}$ is an increasing sequence in $\mathcal{R}$ whose union $E$ is also in $\mathcal{R}$, then $\mu(E)=\operatorname{LUB} \mu\left(E_{n}\right)$.

\subsection{Spatial Interaction}

According to Kingsley and Fotheringham [9], spatial interaction is a broad term encompassing any movement over space that results from a human process. However it can be extend to more than human process, which is involving flow of attribute among two spatial data, for example in our case will be the flow of potential differences among clusters. Among interaction models, the gravity model is the most widely used type of interaction model [9].

\subsection{Gravity Model}

Gravity model is a model that been applied in international trade flow since 1940's [10]. It is a mathematical formulation which utilizes the gravitational force concept, where the Newton's Law of Universal Gravitation states that two body attract each other proportionally to the product of each body's mass divided by the square of the distance between their respective center of gravity.

$$
F_{i j}=G \frac{M_{i} M_{j}}{d_{i j}{ }^{2}}, d_{i j} \neq 0,
$$

meanwhile gravity model is similar with it, which is resultant interaction index between two location is proportionally to the product of attributes of the location and inversely proportional to the distance among the two location. 


$$
T_{i j}=k \frac{P_{i} P_{j}}{d_{i j}}, d_{i j} \neq 0 .
$$

However exponents $\lambda, \alpha$ and $\beta$ are introduced into the model in order to allow for other situations where other variables, aside from the $\mathrm{P}_{\mathrm{i}}, \mathrm{P}_{\mathrm{j}}$ and $d_{i j}$ affect the resultant interaction index [11].

$$
T_{i j}=k \frac{P_{i}^{\lambda} P_{j}^{\alpha}}{d_{i j}^{\beta}}, d_{i j} \neq 0 .
$$

After being introduced by Tinbergen [12], the gravity model was considered to be a useful physical analogy with fortunate empirical validity. Nowaday the model is widely used as an analogy to explain the volume of trade, capital flows, and migration among the countries of the world and widely used to analyse and forecast spatial interaction patterns [11].

\section{RESULTS \& DISCUSSION}

\subsection{Measure Construction}

We introduce some definitions in regards to our works.

Definition 4 (cluster center): Let $\mathbb{C}$ be the set of all the cluster center at time $t$, i.e.: $\mathbb{C}_{t}=\left\{c_{1}, c_{2}, c_{3}, \ldots, c_{m}\right\}$; where $\mathrm{m}=$ number of cluster center at time $\mathrm{t}$. Each cluster carries the position of a flat EEG ( $f$ EEG) and electrical potential, which means that $\left(c_{j}\right)_{t}=\left\{\left(x_{j}, y_{j}\right), v_{j}\right\}_{t}$; where $v_{j}=$ electrical potential of the cluster center $j^{\text {th }}$. Let $\mathbb{P}$ be the entire pixels of fEEG, thus $\mathbb{C}_{t}=\{(x, y), v \mid x, y, v \in \mathbb{P}\}_{t}$ where $\mathbb{P}=\left\{\mathrm{p}_{1}, \mathrm{p}_{2}, \mathrm{p}_{3}, \ldots, \mathrm{p}_{\mathrm{n}}\right\}$ such that $\mathrm{p}_{1}=\left(\mathrm{x}_{\mathrm{i}}, \mathrm{y}_{\mathrm{i}}\right)$, or in short: $\mathbb{C}_{\mathrm{t}} \subset \mathbb{P}$.

Definition 5 (Interaction model): The interaction model is defined by:

$$
T_{i j}=k \frac{v_{i}^{\lambda} v_{j}^{\alpha}}{d_{i j}^{\beta}}, d_{i j} \neq 0 .
$$

such that

$T_{i j}=$ interaction between $i^{\text {th }}$ cluster and $j^{\text {th }}$ cluster.

$d_{i j}=$ Euclid distance between cluster center $i^{t h}$ and $j^{t h}$.

$v_{i}, v_{j}=$ electrical potential of cluster $i^{t h}$ and $j^{\text {th }}$ respectively.

$\lambda, \alpha=$ potential to generate movement and potential to attract movement respectively. It will be the fuzzy membership (in crisp form) of cluster $i^{\text {th }}$ and $j^{\text {th }}$.

$\beta=$ distance decay parameter.

$k=$ rate characteristic. It will be Einstein's constant, which is $k=-\frac{8 \pi G}{c^{2}}=1.87 \times 10^{-27}$.

Definition 6 (Interaction function): $T: \mathbb{P} \times \mathbb{P} \rightarrow \mathbb{R}$ where

$$
T_{i j}=\left\{\begin{array}{l}
k \frac{v_{i}^{\lambda} v_{j}^{\alpha}}{d_{i j}{ }^{\alpha}} \quad ; c_{1} \neq c_{2} \\
0 \quad ; c_{1}=c_{2}, n(A)=0
\end{array}\right.
$$

Remark: Total flow of estimate in a system among all clusters is derived as

$$
\begin{gathered}
T_{\text {total }}=k \frac{v_{c_{1}}{ }^{\lambda}{v_{c_{2}}}^{\alpha}}{d_{c_{1} c_{2}}{ }^{\beta}}+k \frac{v_{c_{1}}{ }^{\lambda}{v_{c_{3}}}^{\alpha}}{d_{c_{1} c_{3}}{ }^{\beta}}+k \frac{v_{c_{1}}{ }^{\lambda}{v_{c_{4}}}^{\alpha}}{d_{c_{1} c_{4}}{ }^{\beta}}+\cdots \\
+k \frac{v_{c_{m-1}}{ }^{\lambda} v_{c_{m}}{ }^{\alpha}}{d_{c_{m-1} c_{m}}{ }^{\beta}}
\end{gathered}
$$

Definition 7 (Domain): The set of cluster interaction on $f$ EEG is defined by:

$$
\begin{gathered}
\mathbb{C}^{2}=\left\{\begin{array}{c}
\left.\left(c_{1}, c_{1}\right),\left(c_{1}, c_{2}\right), \ldots,\left(c_{1}, c_{m}\right),\left(c_{2}, c_{1}\right),\left(c_{2}, c_{2}\right), \ldots\right) \\
\left(c_{2}, c_{m}\right), \ldots,\left(c_{m}, c_{m}\right)
\end{array}\right\} \\
P\left(\mathbb{C}^{2}\right)=\left\{\begin{array}{c}
\emptyset,\left\{\left(c_{1}, c_{1}\right)\right\},\left\{\left(c_{1}, c_{2}\right)\right\}, \ldots,\left\{\left(c_{1}, c_{1}\right),\left(c_{1}, c_{2}\right)\right\}, \\
\left\{\left(c_{1}, c_{1}\right),\left(c_{1}, c_{3}\right)\right\}, \ldots, \mathbb{C}^{2}
\end{array}\right\}
\end{gathered}
$$

Theorem 1: $P\left(\mathbb{C}^{2}\right)$ is a ring of sets.

Proof:

i) For any $A, B \in P\left(\mathbb{C}^{2}\right), A \cup B \subset P\left(\mathbb{C}^{2}\right)$ since all elements of $P\left(\mathbb{C}^{2}\right)$ are subsets (proper or improper) of $\mathbb{C}^{2}$, thus the union of any number of these subsets are closed under $P\left(\mathbb{C}^{2}\right)$.

ii) For any $A \in P\left(\mathbb{C}^{2}\right)$, we know that $A^{C}=\mathbb{C}^{2} \backslash A$, which is proper subsets of $\mathbb{C}^{2}$, and thus $A^{C} \in$ $P\left(\mathbb{C}^{2}\right)$.

By De Morgan's law, $A \cap B=\left(A^{C} \cup B^{C}\right)^{C}$. But $A^{C}$ and $B^{C}$ also in $P\left(\mathbb{C}^{2}\right)$ since $\mathbb{C}^{2} \backslash A$ and $\mathbb{C}^{2} \backslash B$ are proper subset of $\mathbb{C}^{2}$. By (i), $\left(A^{C} \cup B^{C}\right)$ also in $P\left(\mathbb{C}^{2}\right)$. And $\left(A^{C} \cup B^{C}\right)^{C}$ also in $P\left(\mathbb{C}^{2}\right)$.

$$
\therefore A \cap B=\left(A^{C} \cup B^{C}\right)^{C} \in P\left(\mathbb{C}^{2}\right)
$$

Thus $P\left(\mathbb{C}^{2}\right)$ is a ring (of sets).

Theorem 2: Gravity model, $T: P\left(\mathbb{C}^{2}\right) \rightarrow \mathbb{R}$ is a measure. Proof:

(1) Pick any $A, B \in P\left(\mathbb{C}^{2}\right)$. From the remark of "Total flow of estimate in a system among all clusters",

$$
\begin{aligned}
T(A \cup B) & =k \frac{v_{c_{A 1}}{ }^{\lambda} v_{c A_{2}}{ }^{\alpha}}{d_{c_{A 1} c_{A 2}} \beta}+k \frac{v_{c_{B 1}}{ }^{\lambda}{v_{c_{B 2}}}^{\alpha}}{d_{c_{B 1} c_{B 2}} \beta} \\
& =T(A)+T(B)
\end{aligned}
$$

Hence $T$ is additive.

(2) From our model we have 


$$
T_{i j}= \begin{cases}k \frac{v_{i}^{\lambda} v_{j}^{\alpha}}{d_{i j}^{\beta}} & ; c_{1} \neq c_{2}, \\ 0 & ; c_{1}=c_{2},\end{cases}
$$

we know that $k=-\frac{8 \pi G}{c^{2}}=1.87 \times 10^{-27}$ and $\beta=$ 1. Since the direction of current are out of our scope, thus $v_{i}, v_{j} \geq 0$. Besides, $\lambda, \alpha$ are fuzzy membership value that in crisp form, thus $0 \leq$ $\lambda, \alpha \leq 1$. Also Euclid distance is always positive, which $d_{i j} \geq 0$.

Thus we conclude $T_{i j}$ is always positive since $k \frac{v_{i}^{\lambda} v_{j}^{\alpha}}{d_{i j}{ }^{\beta}} \geq 0$.

(3) Observe that $\emptyset \in P\left(\mathbb{C}^{2}\right)$, but then $n(\varnothing)=0$, thus we can say that $T(\varnothing)=0$ (by definition).

(4) Suppose a sequence $\left\{s_{n}\right\}=\left\{s_{1}, s_{2}, s_{3}, \ldots\right\}$ is an increasing sequence in $P\left(\mathbb{C}^{2}\right)$ such that $s_{1} \subset s_{2} \subset$ $s_{3} \subset \cdots \subset s_{n}$. Thus

$$
\begin{aligned}
T\left(s_{2}\right) & =T\left[\left(s_{2}-s_{1}\right) \cup s_{1}\right] \\
& =T\left(s_{2}-s_{1}\right)+T\left(s_{1}\right) \geq T\left(s_{1}\right) \text { since } \\
& T\left(s_{2}-s_{1}\right) \geq 0
\end{aligned}
$$

Thus,

$$
\begin{gathered}
T\left(s_{2}\right) \geq T\left(s_{1}\right) \\
T\left(s_{3}\right) \geq T\left(s_{2}\right) \\
T\left(s_{4}\right) \geq T\left(s_{3}\right) \\
\vdots \\
T\left(s_{n}\right) \geq T\left(s_{n-1}\right)
\end{gathered}
$$

Hence LUB $T\left(\left\{s_{n}\right\}\right)=T\left(s_{n}\right)$. But then

$$
\begin{aligned}
S & =\bigcup_{i=1}^{n} s_{i} \\
& =s_{1} \cup s_{2} \cup s_{3} \cup \ldots \cup s_{n}
\end{aligned}
$$

since $s_{1} \subset s_{2} \subset s_{3} \subset \cdots \subset s_{n}$, we have

$$
S=s_{n}
$$

Therefore $T(S)=T\left(s_{n}\right)=\operatorname{LUB} T\left(\left\{s_{n}\right\}\right)$

Thus $T$ is a measure.

\subsection{Implementation}

A sample of flat-EEG data obtained from [3] is used (Figure 4)(Table 1). The membership value of every cluster center is equal to 1 at $\mathrm{t}=5$ and the distance decay parameter is also assumed as 1 . Note that we did not include those overlapped cluster since the interaction index will be 0 . Also since the spatial interaction index is scalar, the reverse interaction of clusters will not be listed out. The result is presented in Table 2 and Figure 5 to show the relation among distance and spatial interaction index.

From the Figure 5, it shows that the spatial interaction index is increasing in distance from 4.0592 to 4.6758. however it's start to decrease from 4.6758 until 5.6340. After that it become increasing and decrease by turns and hit the maximum spatial interaction index at 8.4817 .

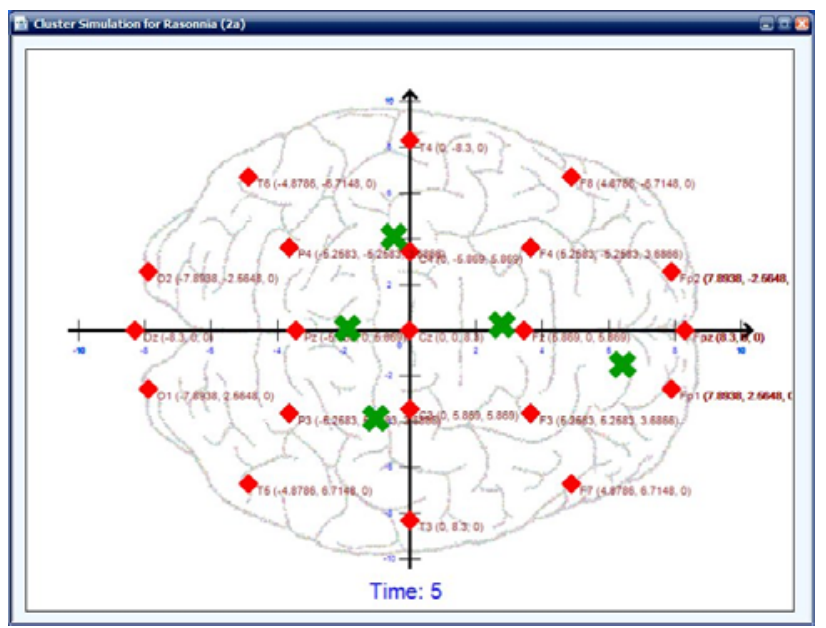

Fig. 4 Flat $\mathrm{EEG}$ at $\mathrm{t}=5$.

Table 1 Five cluster centers with its potential difference for $\mathrm{t}=5$.

\begin{tabular}{|c|c|c|r|}
\hline $\begin{array}{c}\text { Cluster } \\
\text { center }\end{array}$ & $\mathrm{x}$ & $\mathrm{y}$ & \multicolumn{1}{c|}{ Electrical potential } \\
\hline$c_{1}$ & -1.0308 & 3.8862 & 47.401107254677600 \\
\hline$c_{2}$ & 2.7945 & -0.2501 & 4.934408253672210 \\
\hline$c_{3}$ & -0.4832 & -4.1281 & 87.329038079578500 \\
\hline$c_{4}$ & 6.4484 & 1.5180 & 230.553660978112000 \\
\hline$c_{5}$ & -1.8788 & -0.0936 & 129.238901820005000 \\
\hline
\end{tabular}

Table 2 The spatial interaction index of five cluster centers for $\mathrm{t}=5$.

\begin{tabular}{|lccc|}
\hline \multicolumn{2}{|c}{ Cluster to Cluster } & Distance & Spatial Interaction \\
\hline 1 & 2 & 5.6340 & $7.8 \mathrm{E}-26$ \\
& 3 & 8.0330 & $9.64 \mathrm{E}-25$ \\
& 4 & 7.8451 & $2.61 \mathrm{E}-24$ \\
& 5 & 4.0692 & $2.82 \mathrm{E}-24$ \\
\hline 2 & 3 & 5.0776 & $1.59 \mathrm{E}-25$ \\
& 4 & 4.0592 & $5.24 \mathrm{E}-25$ \\
& 5 & 4.6758 & $2.55 \mathrm{E}-25$ \\
\hline 3 & 4 & 8.9401 & $4.21 \mathrm{E}-24$ \\
& 5 & 4.2690 & $4.94 \mathrm{E}-24$ \\
\hline 4 & 5 & 8.4817 & $6.57 \mathrm{E}-24$ \\
\hline \multicolumn{2}{|c}{ Total interaction } & & $2.31 \mathrm{E}-23$ \\
\hline
\end{tabular}




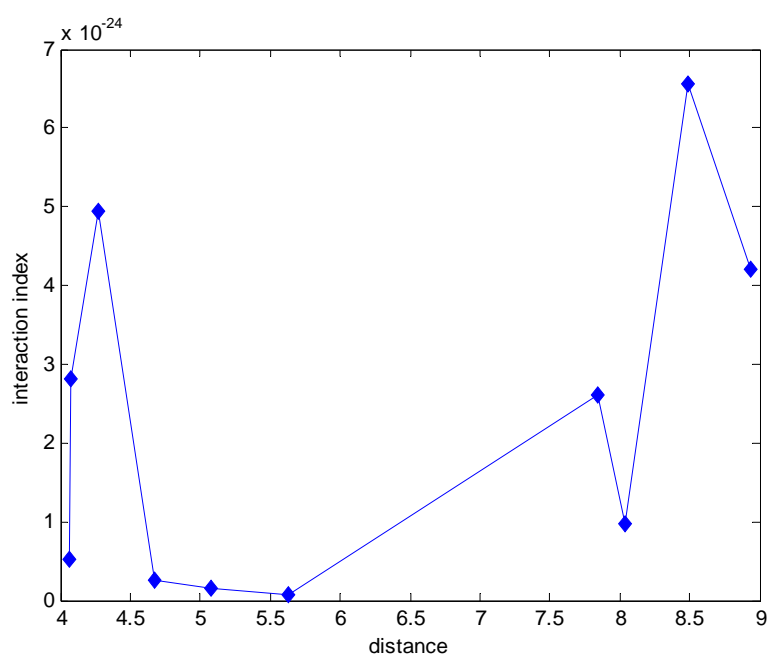

Fig. 5 Graph of distance versus spatial interaction index at time $5^{\text {th }}$

\section{CONCLUSION}

In this paper the gravity model used is proven as a measure. An analysis of cluster centers of an epileptic patient who experienced 10-second seizure was made and at $\mathrm{t}=5$ was chosen as a sample and application of spatial gravity model was applied and analysed.

\section{ACKNOWLEDGEMENT}

The researchers would like to thank the Ministry of Science, Technology and Innovation for funding this research under the Fundamental Research Grant Scheme
(Vot. No. O2H36) and Ministry of Higher Education for granting the MyBrain15(MyPhD) scholarship.

\section{REFERENCES}

[1] F. S. Bao, G. Jue-Ming, H. Jing, D. Y. C. Lie, Z. Yuanlin and K. J. Oommen (2009). Engineering in Medicine and Biology Society, 2009, Annual International Conference of the IEEE., USA, 3-6 Sept. 2009, p.6603-6607.

[2] Sanei, S., \& Chambers, J. A., EEG Signal Processing, Johan Wiley \& Sons Ltd, England, 2007.

[3] Zakaria, F., HPM Modelling of Brain Storm for Epilepsy Sufferers (2007), to Universiti Teknologi Malaysia.

[4] Ahmad, T., Ahmad, R. S., Yun, L. L., Zakaria, F., \& Rahman, W. E. Z. W. A., Matematika 21(1) (2005) 35-42.

[5] Liau, L. Y. Homeomorfisma $\mathrm{S}^{2}$ Antara $\mathrm{E}^{2}$ Melalui Struktur Permukaan Riemann Serta Deduksi Teknik Pembuktiannya Bagi Homeomorfisma Pemetaan Topologi Topografi Kabur (FTTM) (2001), to Universiti Teknologi Malaysia.

[6] Ahmad, T., Abdullah, J., Zakaria, F., Mustapha, F., \& ZabidiHussin, A. Journal of Quantitative Methods, Vol. 2(2) (2006) 1-9.

[7] Hungerford, T. W., Algebra: Graduate Texts in Mathematics. vol. 73 (1980),

[8] S.K. Berberian. Measure and Integration, AMS Chelsea Publishing Series, Amer Mathematical Society, US, 2011.

[9] Haynes, K. E., \& Fotheringham, A. S., Gravity and Spatial Interaction Models: Sage Publications, US, 1985.

[10] Oguledo, V.I. and Macphee, C.R., Applied Economics 26 (1994) 107-120.

[11] A.T.C. Philbrick, B.E., Transportation Gravity Models. University of Queensland. Department of Civil Engineering, Bulletin No. 15December 1971.

[12] Tinbergen, J., Shaping the World Economy: Suggestions for an International Economic Policy. Twentieth Century Fund, New York, 1962 